\title{
Epididymis cholesterol homeostasis and sperm fertilizing ability
}

\author{
Fabrice Saez, Aurélia Ouvrier and Joël R Drevet
}

Cholesterol, being the starting point of steroid hormone synthesis, is a long known modulator of both female and male reproductive physiology especially at the level of the gonads and the impact cholesterol has on gametogenesis. Less is known about the effects cholesterol homeostasis may have on postgonadic reproductive functions. Lately, several data have been reported showing how imbalanced cholesterol levels may particularly affect the post-testicular events of sperm maturation that lead to fully fertile male gametes. This review will focus on that aspect and essentially centers on how cholesterol is important for the physiology of the mammalian epididymis and spermatozoa.

Asian Journal of Andrology (2011) 13, 11-17; doi:10.1038/aja.2010.64; published online 1 November 2010

Keywords: cholesterol homeostasis; epididymosomes; fertility; post-testicular maturation; spermatozoa

\section{INTRODUCTION}

Cholesterol is a central molecule in animal physiology owing to its importance in the maintenance of cell structure, bile salt metabolism and steroid hormone synthesis. When its homeostasis is deregulated, cholesterol may become a deleterious molecule associated with severe pathological situations, such as atherosclerosis and cardiovascular diseases. As the precursor of all sexual steroid hormones, cholesterol has a pivotal function in all areas of male and female reproductive physiology, from sex differentiation to gamete production. This review will focus on the cholesterol functions in a particular aspect of male reproductive physiology that is post-testicular sperm maturation. It has been known for years that maturational events occurring in the female genital tract (that can be grouped under the term 'capacitation') are highly dependent upon sperm plasma membrane cholesterol. ${ }^{1,2} \mathrm{We}$ will emphasize here how cholesterol is also crucial during epididymal preparation of spermatozoa that will then be ready to capacitate.

\section{CHOLESTEROL SYNTHESIS IN THE EPIDIDYMIS}

Although the ability of the epididymal epithelium to produce cholesterol by the process of de novo synthesis has been investigated from the late 1960s and thereafter, data are scarce. Briefly, it was demonstrated that mouse epididymal tissues from caput, corpus and cauda could incorporate $\left[1-{ }^{14} \mathrm{C}\right]$ acetate into cholesterol in an androgen-dependent manner. ${ }^{3}$ The ability to synthesize cholesterol from $\left[1-{ }^{14} \mathrm{C}\right]$ acetate was confirmed a few years later in bovine epididymis. ${ }^{4}$ These authors also showed that the epididymis has an active cholesterol metabolism as cholesterol esters (CEs) were synthesized from either $\left[1-{ }^{14} \mathrm{C}\right]$ acetate or $\left[4-{ }^{14} \mathrm{C}\right]$ cholesterol. Completing these data, it was more recently demonstrated using DNA chips that the key enzymes of the cholesterol synthesis pathway were expressed in the mouse epididymis, including 3-hydroxy-3-methylglutaryl-coenzyme A synthase 1, 3-hydroxy- 3-methylglutaryl-coenzyme A synthase 2 and 3-hydroxy-3-methylglutaryl-coenzyme A reductase 5 (http://mrg.genetics.washington.edu/).

The question raised about the ability of epididymal epithelium to produce de novo synthesized cholesterol is whether it is a precursor for steroid hormone production. This question still does not have a clear answer to date, and epididymal steroid production has been addressed, on the one side, as the capacity to metabolize circulating or testicular steroids and, on the other side, as local steroid synthesis from cholesterol. Androgen metabolism in the epididymis has been demonstrated as early as $1969^{6}$ using in vitro metabolic conversion of androgens to $5 \alpha$-reduced metabolites by cell-free homogenates. Among different tissues, the rat epididymis was described as a major site of this enzymatic activity, ${ }^{7}$ and in vivo injection of tritiated testosterone was shown to lead to the efficient production of dihydrotestosterone (DHT). ${ }^{8}$ Other studies using different strategies have confirmed this function in the rat ${ }^{9,10}$ as well as in other species including ram, ${ }^{11}$ macaque ${ }^{12}$ and mouse. ${ }^{13}$ More recently, the epididymis was shown to be a putative target of the gonadotropic axis, as luteinizing hormone/human chorionic gonadotropin receptors and folliclestimulating hormone receptors were detected in rat epididymal primary cultures. ${ }^{14,15}$ These receptors are, however, involved in estrogens rather than androgen synthesis as these authors showed their roles in the regulation of $17 \beta$-estradiol synthesis. Estrogens have a structural role in the epididymis as morphological changes have been reported in estrogen receptor- $\alpha$ knockout mice. ${ }^{16}$

A particular feature of the epididymal epithelium is that its androgen dependence is related to both circulating androgens and the ones present in the testicular fluid. The active androgen in the epididymal fluid seems to be DHT, coming from the metabolic activity of the epithelium, as its concentration is higher in cauda epididymidis than in rete testis of rat $^{17}$ and bull ${ }^{18}$, and also higher than it is in plasma. 
However, as spermatozoa do not possess the androgen receptor, ${ }^{19}$ the physiological relevance of a high luminal androgen concentration is not elucidated. The first segment of the caput epididymidis is highly sensitive to the luminal content, and it was shown that efferent duct ligation provoked a selective regression of the mouse initial segment. This regression expanded to the proximal caput following castration but then could be reversed by DHT injection. ${ }^{20}$ This study revealed that DHT coming from the testicular fluid was necessary for the murine epididymal initial segment, whereas the other segments were more under the influence of circulating androgens.

Overall, even if the relative functional importance of epididymal cholesterol synthesis versus metabolism is not clear, one can assume that a normal epididymal function, and therefore a normal fertility, relies at least in part on these two parameters.

\section{CHOLESTEROL TRANSPORT AND TRAFFICKING IN THE EPIDIDYMIS}

The ability of the epididymal epithelium to metabolize cholesterol implies that transport mechanisms exist between the blood compartment and the epithelium. The main cholesterol supply for tissues is circulating low-density lipoproteins (LDLs), which are bound in tissues by LDL receptor located in coated pits of the plasma membrane. LDLs are then endocytosed and enter the complex network of endosomal trafficking, a topic that has been reviewed lately by several authors. ${ }^{21,22}$ The intracellular equilibrium between de novo synthesized cholesterol and cholesterol uptake from lipoproteins is ensured in part by cholesterol efflux pathways to extracellular acceptors, usually high-density lipoproteins (HDLs), in a process called reverse cholesterol transport. The efficiency of reverse cholesterol transport relies on the presence of active cholesterol transporters of the ATP-binding cassette $(\mathrm{ABC})$ superfamily in the cell plasma membrane. The physiological importance of these molecules in cholesterol homeostasis has also been reviewed quite recently. ${ }^{23-26}$ The scavenger receptor class B type I (SR-BI) is also present in the cell plasma membrane and is involved in cholesterol transfer from the intracellular compartment to phospholipid-containing acceptors such as HDLs and lipidated apolipoproteins. Cholesterol flux through SR-BI can be bidirectional depending on the concentration gradient. For instance, SR-BI can be used for selective CE uptake from HDLs in steroidogenic tissues such as the adrenal gland ${ }^{27}$ or testicular Leydig cells. ${ }^{28}$ The intracellular concentration of cholesterol is also regulated by an esterification process, and to avoid its toxicity, the produced cholesteryl esters are stored in cytoplasmic lipid droplets.

The importance of these different actors is extensively studied in pathological situations such as atherosclerosis, as reverse cholesterol transport is an atheroprotective process, whereas intracellular CE accumulation is a pathological sign of the arterial wall. The male reproductive function is sensitive to cholesterol homeostasis perturbations, and testicular phenotypes were previously described in knockout mice for nuclear receptors involved in cellular cholesterol homeostasis such as retinoid X receptor- $\beta(\operatorname{RXR} \beta)^{29}$ or liver X receptor- $\beta(\operatorname{LXR} \beta)^{30}$, and also in ABCA1 knockout mice. ${ }^{31}$ In these three mice models, Sertoli cells' CE accumulations lead to several severities of infertility, ranging from a $21 \%$ decrease in $\mathrm{ABCAl}^{-1-}$ mice to complete infertility in RXR $\beta$-null mice. The CE accumulations were shown to be due to an ABCA1-dependent decrease in cholesterol efflux from Sertoli cells in LXR $\beta$-null mice, ABCA1 being a wellknown LXR target gene. ${ }^{32}$

Data are scarce concerning cholesterol homeostasis regulation in the epididymis, but the work by our group on the double-knockout mice for the two liver $\mathrm{X}$ receptors' isoforms $\left(l x r \alpha / \beta^{-/-}\right.$mice) has allowed a better understanding of the situation. The $l \times r \alpha / \beta^{-/-}$males presented an infertility starting at the age of 5 months and a complete sterility when the animals reached the age of 9 months and older. The reproductive failure comes from the association of a testicular phenotype ${ }^{33}$ with an epididymal phenotype. ${ }^{34}$ Out of the five segments constituting the mouse caput epididymidis, the epididymal phenotype observed in $l x r \alpha / \beta^{-/-}$males was specifically located to the first two segments. It was characterized by an enlargement of the tubule lumen, the accumulation of an amorphous substance in the lumen and shrinkage of the epithelial height. This phenotype was not secondary to the decrease in plasma testosterone, as a 15-day supplementation did not restore the normal epididymis in 7.5- and 10.5-month-old animals. The epithelial function was also altered, as decreases in specific epididymal gene expression were noticed (glutathione peroxidase-5 and Ets-like polyoma-enhancer activator 3 trans-acting factor). Lipid accumulations, revealed by oil red $\mathrm{O}$ staining, were present in the peritubular and interstitial tissues as well as in the epithelium of $l x r \alpha / \beta^{-1-}$ male mice. The amorphous substance in the tubule lumen was not stained, it was thus not of lipidic origin. Spermatozoa were also shown to be fragile as many isolated heads and flagella were observed when sperms were retrieved from the cauda epididymidis, which might be the result of both testicular and epididymal dysfunctions. This phenotype was further characterized in a more recent study ${ }^{35}$ showing that neutral lipids revealed by oil red O staining were cholesteryl esters. More precisely, cholesterol trafficking was shown to be an LXR-regulated mechanism in mice caput epididymidis in a segment- and cell-specific manner. ${ }^{36}$ In $l \times r \alpha / \beta^{-/-}$ animals, only a particular cell type of the first two caput segments, the apical cells, showed CE accumulations in their cytoplasm, associated with a loss of the membrane cholesterol transporter ABCA1 and the triggering of the apoptotic pathway (as shown via terminal deoxynucleotidyl transferase dUTP nick end labeling detection assay). These data confirmed the important role of ABCA1 in the male reproductive tract, as it was shown elsewhere that male mice invalidated for this gene had a $21 \%$ decrease in their fertility over their lifespan. Indeed, when crossed with wild-type females, $\mathrm{ABCA1}^{-1-}$ males aged 4-5, 7-8 and 11-13 months gave fewer litters (taking into account females with copulatory plugs) compared with wild-type males. ${ }^{31}$ This phenotype was associated with a decrease in intratesticular testosterone levels and sperm counts.

Our work also showed that the expression and location of another $\mathrm{ABC}$ cholesterol transporter, ABCG1, was different from ABCA1, and was not altered in the epididymides of $l x r \alpha / \beta^{-/-}$male mice. More precisely, ABCG1 was present at the apical pole of all epithelial cells in the proximal caput epididymal segments, ${ }^{36}$ suggesting complementary functions for these two cholesterol transporters in the epididymal epithelium, as previously shown in vitro. ${ }^{37}$ These locations also favor the hypothesis of cholesterol efflux from the epithelium to the lumen, to interact with and mature the transiting sperm. This raises the question of which acceptors are present in the epididymal fluid, a delicate point to address in the mouse model. Interesting data have been published of late showing the presence of several $\mathrm{ABC}$ transporters (ABCA1, ABCA7 and ABCG1) on mouse sperm cells collected from the caput epididymidis. ${ }^{38}$ ABCA17 had also previously been detected in the anterior head of sperm and elongated spermatids of the mouse and rat. ${ }^{39}$ These $\mathrm{ABC}$ transporters present on the sperm plasma membrane may be involved in the cholesterol fluxes occurring during sperm epididymal maturation, and their role could also be important in the regulation of the capacitation process, two points that will be 
developed later. The putative function of ABCA17, as shown in HEK293 cell cultures, is to efflux CEs, triacylglycerols and fatty acid esters. It may thus be more active during sperm production in the testis if it has the same functional properties. ${ }^{39}$

Cholesterol homeostasis defects were also shown in male mice deficient in the acid sphingomyelinase, a lysosomal hydrolase for which mutations are at the origin of types A and B Niemann-Pick diseases. Niemann-Pick diseases are neurodegenerative diseases (the type A being severe) characterized by lipid storage disorders, mainly owing to the accumulation of sphingomyelin (SM) and cholesterol, in the cells and tissues of the patients. In this model, the caput and cauda epididymal epithelial cells presented lipid-filled vacuoles and caudaretrieved spermatozoa had elevated levels of SM and cholesterol, leading to disrupted plasma and acrosome membrane, morphology and motility defects as well as abnormal capacitation. ${ }^{40}$ This model established a link between cholesterol homeostasis defects and post-testicular maturation defects as the phenotype was partly because of testicular defects and partly of epididymal alterations.

The importance of lipoproteins in fertility was demonstrated as knockout mice for type 2 apolipoprotein E receptor (apoER2), a member of the LDL receptor gene family, showed a phenotype of male infertility. ${ }^{41}$ ApoER2 is highly expressed in the epithelium of the initial segment of the epididymis and males were infertile owing to the inability of sperms to regulate their cell volume, leading to abnormal sperm morphology and immotility. Clusterin was identified as an endogenous ligand for apoER2 by the same authors; however, the invalidation of the clusterin gene has only a slight effect on fertility. ${ }^{42}$ The molecular pathways involved thus need further investigation but could yield new insights into the concept of lipid-related sperm maturation.

The presence of SR-BI in the mouse epididymidis was published by our group, ${ }^{36}$ and we showed, using immunohistochemical detection, that SR-BI is strongly present at the apical pole of the epididymal epithelium in the proximal caput. The staining intensity revealed strong differences among the different segments, suggesting a regulation along the duct. We could also observe a peritubular location of SR-BI in the smooth muscle cells that was constant along the tubule. More peculiar was the observation of a redistribution of SR-BI from an apical location to intercellular lateral membranes, from the end of the caput down to the cauda epididymidis (data not shown). The significance of this precise distribution is not known yet, but the presence of lipoprotein receptors once again in the proximal epididymis (as noticed for apoER2) suggests that cholesterol movements are functionally important in this part of the organ. Mouse model invalidated for SR-BI have been generated, and only female infertility was reported by the authors. ${ }^{43}$ This may suggest a functional redundancy between the different cholesterol transporters seen in the epididymal epithelium.

Another model, the male mice heterozygous for a targeted mutation of the apolipoprotein $\mathrm{B}(a p o B)$ gene, confirmed the role of apolipoproteins in male fertility. Indeed, these males exhibited severely compromised fertility, mainly because of failure of the sperm cells to fertilize eggs both in vivo and in vitro. ${ }^{44,45}$ This was accompanied by reduced sperm motility, survival time and sperm count, also contributing to the infertility phenotype. The specific role of apoB in this phenotype was confirmed by the fact that the introduction of the genomic sequence encoding human apoB into these animals restored normal fertility. The authors did not observe an epididymal origin to explain the observed abnormalities. However, without knowing the site of apoB expression, they could not conclude on whether the phenotype was a consequence of a systemic perturbation or was related to testis and/or epididymis-restricted alterations.
Overall, these data show that apolipoproteins and their receptors are key players for male fertility both in the testis during gametogenesis and also in the epididymis. Cholesterol homeostasis thus needs to be highly regulated in the male epididymal duct, probably to ensure a proper post-testicular maturation of the male gametes. Clearly, more research in this field is needed to better understand the precise roles played by the apolipoproteins and the underlying mechanisms related to sperm cell maturation.

Considering the role played by cholesterol homeostasis in the male reproductive organs, one can wonder whether dietary cholesterol overload could have negative consequences on male fertility. Hypercholesterolemic rats show decreases in fertility index, testicular weight, sperm cell count, sperm motility and viability, and an increase in sperm cell abnormalities. ${ }^{46}$ These parameters were attributed mainly to testicular degeneration that could be overcome by simvastatin treatment. Another study on hypercholesterolemic rats revealed that epithelial cell height was reduced in caput and cauda epididymides, along with an alteration of the seminiferous tubules in the testes. ${ }^{47}$ Metabolic syndrome is a highly prevalent pathology in Western countries, with, for example, 47 million US residents affected. One of the criteria characterizing this pathology is dyslipidemia and it was shown that among 106 male partners from infertile couples, there was a $65 \%$ incidence of dyslipidemia as defined by isolated hypercholesterolemia, hypertriglyceridemia or both. ${ }^{48}$ Metabolic syndrome is a multifaceted pathology with obesity, dyslipidemia, hypertension and insulin resistance. The incidence of these parameters on male fertility was reviewed lately. ${ }^{49}$ The importance of epididymal sperm maturation is often considered as secondary compared with testicular defects and endocrine perturbations associated with dyslipidemia, obesity or hypercholesterolemia. We, very recently, observed that 3-month-old $l \times r \alpha / \beta^{-/-}$male mice, which are totally fertile at this young age, once fed for 4 weeks with a $1.25 \%$ cholesterol-containing diet became totally infertile without showing any testicular alteration. The infertility was because of proximal epididymis destructuration in an atherosclerosis-like manner, leading to sperm morphological abnormalities, decreased motility and viability, and premature acrosome reaction (Ouvrier et al., unpublished). This study revealed that the epididymis is an early target of cholesterol toxicity in this dyslipidemic mouse model, and raised the question of the importance of post-testicular sperm alterations in dyslipidemia-associated male infertility, a topic largely uncovered in many studies.

\section{EPIDIDYMAL SPERM MATURATION AND CHOLESTEROL HOMEOSTASIS}

As a consequence of gamete production and maturation, sperm cells stored in the cauda epididymidis must possess the biochemical, structural and functional properties to fulfill the last steps of the fertilization process in the female genital tract, that is, the capacitation, the binding of the zona pellucida and the acrosome reaction to finally fuse with the oocyte.

The modification of the sperm cholesterol content during epididymal maturation has been investigated in several mammals, and a significant decrease of about $50 \%$ has been reported in ram, ${ }^{50}$ rat $^{51}$ hamster ${ }^{52}$ and mouse. ${ }^{53}$ This cholesterol loss is usually accompanied by a decrease in the cholesterol/phospholipids ratio, an indicator of membrane fluidity, thus suggesting that sperm cells increase their membrane fluidity when descending the epididymis, probably to be able to undergo the fusion steps in the female genital tract. In one species, the goat, however, the sperm cholesterol content increases during epididymal maturation, ${ }^{54}$ whereas in boar, no significant 
changes were noticed. ${ }^{55}$ In human spermatozoa, using membrane anisotropy measurement by fluorescence polarization, the fluidity of cauda epididymal sperm was shown to be higher than the one from caput epididymal sperm. ${ }^{56}$

A particularity of the sperm cells in their sterol composition is the high proportion of desmosterol that was first reported in hamster spermatozoa. ${ }^{57}$ Desmosterol is a cholesterol precursor, usually present in certain tissues such as brain as a transitory cholesterol form. Desmosterol has a double bond at C24, which is reduced by a desmosterol reductase (DHCR24, 3 $\beta$-hydroxysteroid- $\Delta^{24}$-reductase) to form cholesterol. Surprisingly, mice invalidated for this enzyme showed no important morphological defects and survived to full term. However, they showed strong disturbances in sterol homeostasis, with $99 \%$ of plasma and tissue sterols being desmosterol. ${ }^{58,59}$ The functional importance of cholesterol in reproductive physiology is sustained by the fact that these mice are unable to reproduce, the males showing degenerated testes, comparable to what is obtained following a treatment with a chemical inhibitor of DHCR $24 .{ }^{60}$ Unfortunately, to our knowledge, no data are available regarding the epididymal structure of these mice. The relative proportion of sperm desmosterol among total sterols is high in several species and also changes during epididymal transit, with an increase in desmosterol proportions reported in boar. ${ }^{55}$ The ratio to cholesterol was further investigated and was shown to increase from trace amounts in hamster caput epididymal sperm to 0.45 in cauda epididymal sperm, ${ }^{52}$ and from 0.11 to 0.76 in rat sperm. ${ }^{61}$ Desmosterol represents $59 \%$ of total sterols in rhesus monkey sperm ${ }^{62}$ and $25 \%$ in ejaculated human sperm. ${ }^{63}$

The physiological significance of this high proportion of desmosterol in mammalian sperm cells is largely unknown. Some works have reported the selective presence of desmosterol in primate sperm flagella, thus putatively involving it in the regulation of sperm movement. ${ }^{64}$ Others have shown that desmosterol was involved, like cholesterol, in the regulation of the human sperm capacitation process. ${ }^{65,66}$ The loss of these molecules from the sperm plasma membrane in the female genital tract is an early event of the capacitation process that we will discuss thereafter. On a clinical point of view, the cholesterol/phospholipids ratio was found to be higher in sperm cells from patients with idiopathic infertility ${ }^{67}$ and it was also demonstrated that the cholesterol/desmosterol ratio could be related to male fertility. A recent report showed a positive correlation between cholesterol/desmosterol values and sperm concentrations, sperm motility, linear velocity, normal sperm morphology and acrosome reaction percentage in human sperm fractions separated by density gradient centrifugation. ${ }^{68}$ Furthermore, these authors showed that the above-cited parameters are negatively correlated with the cholesterol/phospholipids ratio, thus emphasizing that human sperm functions are dependent at least in part on their sterol composition. Other sterols such as cholesterol sulfate or cholesta-7,24-dien-3 $\beta$-ol have also been described in mammalian sperm, but their functional relevance has always remained obscure.

Even if this is not the topic of this review, it is important to mention here that the fatty acid composition of the sperm cells is also highly modified during the epididymal transit, with an increase in the relative proportion of polyunsaturated fatty acids, another important factor contributing to the membrane fluidity of these cells. The importance of these fatty acids in the female and male reproductive function has been reviewed of late ${ }^{69}$ and there is a link between dietary intake and sperm properties. The molecular mechanisms underlying sperm fatty acid maturation in the epididymis are largely unknown, as the ones regulating cholesterol maturation. Future studies will have to be conducted to better understand these mechanisms and whether they are related or independent, as human fertility is highly challenged by the perturbations coming from the dietary intakes. An emerging idea in the last few years is that the testis may not be the sole target for metabolic perturbations. Altered post-testicular maturation steps may have strong consequences on the fertility outcome, for natural conception as well as medically assisted reproduction. ${ }^{70,71}$

Biochemical modifications of sterols and fatty acids occurring in the epididymis have a direct influence on the sperm plasma membrane architecture and dynamics ${ }^{72}$. Different biophysical methods can be used to investigate the membrane dynamics of sperm plasma membranes, all presenting limits in their accuracy and discrimination capacity between the subdomains of this particular cell. The aim of this review is not to make a state-of-the-art concerning these methods, but to outline the relation between cholesterol epididymal sperm maturation and sperm function. We will thus focus on the functional links existing between sperm structure and fertilizing power in the following section. The regionalization of membrane cholesterol was shown in mouse sperm using filipin staining, with more cholesterol/filipin complexes present in the acrosomal region, whereas the post-acrosomal region was weakly labeled. ${ }^{73}$ The changes in membrane structure were verified in ram spermatozoa for which fluidity increased between testis and cauda epididymidis, whereas no significant changes were measured in boar, a species known for showing only few lipid maturations during epididymal passage. ${ }^{74}$ It was confirmed that the diffusion coefficient of fluorescent lipid reporter probes, indicating the fluidity of the membrane, was three to four times superior in the acrosome than in other parts of the cells (principal piece of the tail) and it increased during epididymal maturation in mouse sperm. Cholesterol diffusion was higher in the sperm head than in the tail, and showed heterogeneous distribution when detected with filipin. ${ }^{75}$ These authors also confirmed the above data in ram, goat, dog and monkey sperms. ${ }^{76}$ The particular structure of the plasma membrane overlying the acrosome was confirmed in live mouse sperm, where ganglioside M1, a marker of lipid rafts, that is membrane subdomains enriched in sterols and sphingolipids, appeared selectively located in this sperm structure, as detected by fluorescent-labeled cholera toxin subunit B. ${ }^{77}$ These data were confirmed in human sperm by the same group, ${ }^{78}$ and they also showed that the subacrosomal ring (a dense cytoskeletal structure at the base of the plasma membrane over the acrosome) could be considered as a specialized diffusion barrier in the sperm head plasma membrane of living cells only, this barrier becoming non-functional upon cell death. This statement has its importance when studying sperm cells, suggesting that structural approaches should only be undertaken on living cells and not on fixed sperm. Mouse sperm membrane microarchitecture is composed of three different types of membrane domains, or rafts, obtained by separation on a density gradient without the use of detergent. ${ }^{79}$ Their characterization has revealed that one subtype is enriched in sterols, one is enriched in ganglioside M1 and the third one is enriched in both. ${ }^{79}$ These recent data set new bases for the analysis of sperm membrane dynamics during the last steps of fertilization, but also raise the question of whether epididymal maturation has a role in the settings of these properties, given the known changes occurring when spermatozoa descend the epididymal tubule.

The particular membrane composition and architecture acquired by sperm cells between their production in the testis and their storage in the cauda epididymidis finds its high relevance in the maturational events occurring in the female genital tract. It has been clearly shown that cholesterol efflux from the sperm plasma membrane triggers 
signal transduction pathways regulating the capacitation process (reviewed in Ref. 80). This efflux is associated with changes in sperm membrane dynamics and structure, allowing the last fertilization step to occur. ${ }^{81,82}$ The reader is referred to the above-cited articles for a complete covering of this topic.

\section{MECHANISMS OF SPERM PLASMA MEMBRANE CHOLESTEROL MATURATION: HYPOTHESES AND MODELS}

Even if it is well admitted that sperm plasma membrane cholesterol content changes during epididymal maturation of most mammalian males, influencing the structure and dynamics of this membrane, the molecular events by which these changes occur are still largely unknown. It has been demonstrated that modifying the fatty acid dietary intake modifies the fatty acid composition of the sperm plasma membrane in rabbit, ${ }^{83}$ boar $^{84,85}$ and also in chicken, ${ }^{86}$ but such demonstration remains to be done for cholesterol. However, these results clearly suggest that transport mechanisms are active between circulating lipids and epididymal lumen. The presence of different lipoprotein receptors and cholesterol transmembrane transporters in different epididymal cellular subtypes also strengthens this hypothesis. An interesting point is that the epididymal epithelium uses a particular secretion pathway that is the apocrine secretion. ${ }^{82,87}$ It consists of the secretion of small membranous vesicles by the principal cells in the epididymal lumen, called epididymosomes, and this mode has been shown in several species including human, ${ }^{88}$ mouse, ${ }^{53}$ sheep, ${ }^{89}$ rat, ${ }^{90}$ hamster ${ }^{91}$ and bull. ${ }^{92}$ Epididymosomes have a particular lipid composition with a high proportion of SM in the mouse $\mathrm{s}^{53}$ and a cholesterol/phospholipids ratio varying among species from 0.5 in the mouse ${ }^{53}$ to around 2 in bovine. ${ }^{93}$ Mouse epididymosomes were shown to contain high proportions of SM and arachidonic acid. During epididymal transit, the proportion of SM and the cholesterol/phospholipids ratio increased markedly, associated with a decrease in their fluidity measured by fluorescence anisotropy. ${ }^{53}$ These changes are the opposite of the ones described for maturing sperm cells, thus suggesting that epididymosomes could have an important role in the lipid maturation process of epididymal spermatozoa. Furthermore, the transfer of glycosylphosphatidylinositolanchored proteins was demonstrated between lipid rafts of bovine epididymosomes and lipid rafts on sperm plasma membrane. ${ }^{94}$ $\mathrm{P} 25 \mathrm{~b}$, one of the transferred proteins, is the bovine ortholog of $\mathrm{P} 34 \mathrm{H}$, which was shown to be a male fertility marker in humans. ${ }^{95,96}$

The complete mammalian sperm epididymal maturation thus results from mechanisms for lipid and protein changes that are very likely interdependent. The exact role(s) of epididymosomes in the lipid maturation still remains to be investigated and does not exclude the participation of other lipid vesicles subtypes, but will be a challenging research topic.

\section{CONCLUSION}

The regulation of cholesterol homeostasis in the epididymis is a critical point to ensure the normal function of the male gametes in the last steps of the fertilization process. This subject needs further investigations, in complement of the research made on the testis, and this field of research will without any doubt bring new insights in the understanding of the complex outcome of male infertility, a point emphasized by the growing proportion of males in their reproductive age, with dyslipidemia, having difficulties to conceive.

\section{COMPETING FINANCIAL INTERESTS}

The authors declare no competing financial interests.
1 Davis BK, Byrne R, Hungund B. Studies on the mechanism of capacitation. II. Evidence for lipid transfer between plasma membrane of rat sperm and serum albumin during capacitation in vitro. Biochim Biophys Acta 1979; 558: 257-66.

2 Abou-haila A, Tulsiani DR. Signal transduction pathways that regulate sperm capacitation and the acrosome reaction. Arch Biochem Biophys 2009; 485: 72-81.

3 Hamilton DW, Jones AL, Fawcett DW. Cholesterol biosynthesis in the mouse epididymis and ductus deferens: a biochemical and morphological study. Biol Reprod 1969; 1: 167-84.

4 Evans RR, Johnson AD. The metabolic activity of the bovine epididymis. III. Cholesterol and esterified cholesterol metabolism. J Reprod Fertil 1975; 43: 527-30.

5 Johnston DS, Jelinsky SA, Bang HJ, DiCandeloro P, Wilson E et al. The mouse epididymal transcriptome: transcriptional profiling of segmental gene expression in the epididymis. Biol Reprod 2005; 73: 404-13.

6 Inano H, Machino A, Tamaoki B. In vitro metabolism of steroid hormones by cell-free homogenates of epididymides of adult rats. Endocrinology 1969; 84: 997-1003.

7 Gloyna RE, Wilson JD. A comparative study of the conversion of testosterone to 17 beta-hydroxy-5-alpha-androstan-3-one (Dihydrotestosterone) by prostate and epididymis. J Clin Endocrinol Metab 1969; 29: 970-7.

8 Djoseland O, Hansson V, Haugen HN. Androgen metabolism by rat epididymis. 1. Metabolic conversion of ${ }^{3} \mathrm{H}$-testosterone in vivo. Steroids 1973; 21 : 773-83.

9 Kumari GL, Allag IS, Das RP, Datta JK. Regional differences in steroidogenesis and hormone levels in the epididymis and vas deferens of adult rats. Int J Androl 1980; 3: 267-81.

10 Wiszniewska B. Steroidogenic characteristics of in vitro cultured epididymal epithelia cells of the rat. Reprod Biol 2001; 1: 60-6.

11 Amann RP, Marengo SR, Brown DV. Steroidogenesis and testosterone metabolism in cultured principal cells from the ram epididymis. J Androl 1987; 8: 238-46.

12 Simard J, Melner MH, Breton N, Low KG, Zhao HF et al. Characterization of macaque 3 beta-hydroxy-5-ene steroid dehydrogenase/delta 5-delta 4 isomerase: structure and expression in steroidogenic and peripheral tissues in primate. Mol Cell Endocrinol 1991; 75: 101-10.

13 Jean-Faucher C, Berger M, Gallon C, de Turckheim M, Veyssiere G et al. Regional differences in the testosterone to dihydrotestosterone ratio in the epididymis and vas deferens of adult mice. J Reprod Fertil 1986; 76: 537-43

14 Awider-Al-Amawi M, Marchlewicz M, Kolasa A, Wenda-Rozewicka L, Wiszniewska B. Rat epididymal epithelial cells and 17 beta-estradiol synthesis under hCG stimulation in vitro. Folia Histochem Cytobiol 2007; 45: 255-63.

15 Swider-Al-Amawi M, Kolasa A, Sikorski A, Marchlewicz M, Baranowska-Bosiacka I et al. The immunoexpression of FSH-R in the ductuli efferentes and the epididymis of men and rat: effect of FSH on the morphology and steroidogenic activity of rat epididymal epithelial cells in vitro. J Biomed Biotechnol 2010; 2010: 506762.

16 Hess RA, Bunick D, Lubahn DB, Zhou Q, Bouma J. Morphologic changes in efferent ductules and epididymis in estrogen receptor-alpha knockout mice. J Androl 2000; 21: 107-21.

17 Turner TT, Jones CE, Howards SS, Ewing LL, Zegeye B et al. On the androgen microenvironment of maturing spermatozoa. Endocrinology 1984; 115: 1925-32.

18 Ganjam VK, Amann RP. Steroids in fluids and sperm entering and leaving the bovine epididymis, epididymal tissue, and accessory sex gland secretions. Endocrinology 1976; 99: 1618-30.

19 Turner TT. Necessity's potion: inorganic ions and small organic molecules in the epididymal lumen. In: Robaire B, Hinton BT, editors. The Epididymis: From Molecules to Clinical Practice. A Comprehensive Survey of the Efferent Ducts, the Epididymis and the Vas Deferens. New York: Kluwer Academic/Plenum Publishers; 2002. pp131-50.

20 Avram C, Yeung CH, Nieschlag E, Cooper TG. Regulation of the initial segment of the murine epididymis by dihydrotestosterone and testicular exocrine secretions studied by expression of specific proteins and gene expression. Cell Tissue Res 2004; 317 13-22.

21 Ikonen E. Cellular cholesterol trafficking and compartmentalization. Nat Rev Mol Cell Biol 2008; 9: 125-38.

22 Chang TY, Chang CC, Ohgami N, Yamauchi Y. Cholesterol sensing, trafficking, and esterification. Annu Rev Cell Dev Biol 2006; 22: 129-57.

23 Baldan A, Tarr P, Lee R, Edwards PA. ATP-binding cassette transporter $\mathrm{G}_{1}$ and lipid homeostasis. Curr Opin Lipidol 2006; 17: 227-32.

24 Oram JF, Vaughan AM. ATP-binding cassette cholesterol transporters and cardiovascular disease. Circ Res 2006; 99: 1031-43.

25 Sporstol M, Mousavi SA, Eskild W, Roos N, Berg T. ABCA1, ABCG1 and SR-BI: hormonal regulation in primary rat hepatocytes and human cell lines. $B M C \mathrm{Mol} B i 0$ 2007; 8: 5.

26 Tall AR, Yvan-Charvet L, Terasaka N, Pagler T, Wang N. HDL, ABC transporters, and cholesterol efflux: implications for the treatment of atherosclerosis. Cell Metab2008, 7: 365-75.

27 Temel RE, Trigatti B, DeMattos RB, Azhar S, Krieger M et al. Scavenger receptor class $\mathrm{B}$, type I (SR-BI) is the major route for the delivery of high density lipoprotein cholesterol to the steroidogenic pathway in cultured mouse adrenocortical cells. Proc Natl Acad Sci USA 1997; 94: 13600-5

28 Landschulz KT, Pathak RK, Rigotti A, Krieger M, Hobbs HH. Regulation of scavenger receptor, class $\mathrm{B}$, type $\mathrm{I}$, a high density lipoprotein receptor, in liver and steroidogenic tissues of the rat. J Clin Invest 1996; 98: 984-95 
29 Kastner P, Mark M, Leid M, Gansmuller A, Chin W et al. Abnormal spermatogenesis in RXR beta mutant mice. Genes Dev 1996; 10: 80-92.

30 Robertson KM, Schuster GU, Steffensen KR, Hovatta O, Meaney S et al. The liver X receptor-beta is essential for maintaining cholesterol homeostasis in the testis. Endocrinology 2005; 146: 2519-30.

31 Selva DM, Hirsch-Reinshagen V, Burgess B, Zhou S, Chan J et al. The ATP-binding cassette transporter 1 mediates lipid efflux from Sertoli cells and influences male fertility. J Lipid Res 2004; 45: 1040-50.

32 Repa JJ, Turley SD, Lobaccaro JA, Medina J, Li L et al. Regulation of absorption and ABC1-mediated efflux of cholesterol by RXR heterodimers. Science 2000; 289: 1524-9.

33 Volle DH, Mouzat K, Duggavathi R, Siddeek B, Dechelotte P et al. Multiple roles of the nuclear receptors for oxysterols liver $\mathrm{X}$ receptor to maintain male fertility. $\mathrm{Mol}$ Endocrinol 2007; 21: 1014-27.

34 Frenoux JM, Vernet P, Volle DH, Britan A, Saez F et al. Nuclear oxysterol receptors, LXRs, are involved in the maintenance of mouse caput epididymidis structure and functions. J Mol Endocrinol 2004; 33: 361-75.

35 Saez F, Chabory E, Cadet R, Vernet $\mathrm{P}$, Baron $\mathrm{S}$ et al. Liver X receptors and epididymal epithelium physiology. Asian J Androl 2007; 9: 574-82.

36 Ouvrier A, Cadet R, Vernet $\mathrm{P}$, Laillet $\mathrm{B}$, Chardigny JM et al. LXR and ABCA1 contro cholesterol homeostasis in the proximal mouse epididymis in a cell-specific manner. $J$ Lipid Res 2009; 50: 1766-75.

37 Vaughan AM, Oram JF. ABCA1 and ABCG1 or ABCG4 act sequentially to remove cellular cholesterol and generate cholesterol-rich HDL. J Lipid Res 2006; 47: 2433-43.

38 Morales $\mathrm{CR}$, Marat AL, Ni X, Yu Y, Oko R et al. ATP-binding cassette transporters $A B C A 1, A B C A 7$, and ABCG1 in mouse spermatozoa. Biochem Biophys Res Commun 2008; 376: 472-7

39 Ban N, Sasaki M, Sakai H, Ueda K, Inagaki N. Cloning of ABCA17, a novel rodent sperm-specific ABC (ATP-binding cassette) transporter that regulates intracellular lipid metabolism. Biochem J 2005; 389: 577-85.

40 Butler A, He X, Gordon RE, Wu HS, Gatt S et al. Reproductive pathology and sperm physiology in acid sphingomyelinase-deficient mice. Am J Pathol 2002; 161: 1061-75.

41 Andersen OM, Yeung $\mathrm{CH}$, Vorum H, Wellner M, Andreassen TK et al. Essential role of the apolipoprotein E receptor-2 in sperm development. J Biol Chem 2003; 278: 23989-95.

42 Bailey RW, Aronow B, Harmony JA, Griswold MD. Heat shock-initiated apoptosis is accelerated and removal of damaged cells is delayed in the testis of clusterin/ApoJ knock-out mice. Biol Reprod 2002; 66: 1042-53.

43 Trigatti B, Rayburn H, Vinals M, Braun A, Miettinen $\mathrm{H}$ et al. Influence of the high density lipoprotein receptor SR-BI on reproductive and cardiovascular pathophysiology. Proc Natl Acad Sci USA 1999; 96: 9322-7.

44 Huang LS, Voyiaziakis E, Markenson DF, Sokol KA, Hayek T et al. apo B gene knockout in mice results in embryonic lethality in homozygotes and neural tube defects, male infertility, and reduced HDL cholesterol ester and apo A-I transport rates in heterozygotes. J Clin Invest 1995; 96: 2152-61.

45 Huang LS, Voyiaziakis E, Chen HL, Rubin EM, Gordon JW. A novel functional role for apolipoprotein B in male infertility in heterozygous apolipoprotein B knockout mice. Proc Natl Acad Sci USA 1996; 93: 10903-7.

46 Shalaby MA, el-Zorba HY, Kamel GM. Effect of alpha-tocopherol and simvastatin on male fertility in hypercholesterolemic rats. Pharmacol Res 2004; 50: 137-42.

47 Bataineh HN, Nusier MK. Effect of cholesterol diet on reproductive function in male albino rats. Saudi Med J 2005; 26: 398-404.

48 Ramirez-Torres MA, Carrera A, Zambrana M. High incidence of hyperestrogenemia and dyslipidemia in a group of infertile men. Ginecol Obstet Mex 2000; 68: 224-9. Spanish.

49 Kasturi SS, Tannir J, Brannigan RE. The metabolic syndrome and male infertility. J Androl 2008; 29: 251-9.

50 Parks JE, Hammerstedt RH. Development changes occurring in the lipids of ram epididymal spermatozoa plasma membrane. Biol Reprod 1985; 32: 653-68.

51 Aveldano MI, Rotstein NP, Vermouth NT. Lipid remodelling during epididymal maturation of rat spermatozoa. Enrichment in plasmenylcholines containing longchain polyenoic fatty acids of the n-9 series. Biochem J 1992; 283 (Pt 1): 235-41.

52 Awano M, Kawaguchi A, Mohri H. Lipid composition of hamster epididymal spermatozoa. J Reprod Fertil 1993; 99: 375-83.

53 Rejraji H, Sion B, Prensier G, Carreras M, Motta C et al. Lipid remodeling of murine epididymosomes and spermatozoa during epididymal maturation. Biol Reprod 2006; 74: 1104-13.

54 Rana AP, Majumder GC, Misra S, Ghosh A. Lipid changes of goat sperm plasma membrane during epididymal maturation. Biochim Biophys Acta 1991; 1061: 185-96

55 Nikolopoulou M, Soucek DA, Vary JC. Changes in the lipid content of boar sperm plasma membranes during epididymal maturation. Biochim Biophys Acta 1985; 815: 486-98.

56 Haidl G, Opper C. Changes in lipids and membrane anisotropy in human spermatozoa during epididymal maturation. Hum Reprod 1997; 12: 2720-3.

57 Bleau G, VandenHeuvel WJ. Desmosteryl sulfate and desmosterol in hamster epididymal spermatozoa. Steroids 1974; 24: 549-56.

58 Wechsler A, Brafman A, Shafir M, Heverin M, Gottlieb H et al. Generation of viable cholesterol-free mice. Science 2003; 302: 2087
59 Heverin M, Meaney S, Brafman A, Shafir M, Olin M et al. Studies on the cholesterolfree mouse: strong activation of LXR-regulated hepatic genes when replacing cholesterol with desmosterol. Arterioscler Thromb Vasc Biol 2007; 27: 2191-7.

60 Singh SK, Chakravarty S. Antispermatogenic and antifertility effects of 20,25diazacholesterol dihydrochloride in mice. Reprod Toxicol 2003; 17: 37-44.

61 Lindenthal B, Aldaghlas TA, Kelleher JK, Henkel SM, Tolba R et al. Neutral sterols of rat epididymis. High concentrations of dehydrocholesterols in rat caput epididymidis. J Lipid Res 2001; 42: 1089-95.

62 Lin DS, Connor WE, Wolf DP, Neuringer M, Hachey DL. Unique lipids of primate spermatozoa: desmosterol and docosahexaenoic acid. J Lipid Res 1993; 34: 491-9.

63 Sion B, Grizard G, Boucher D. Quantitative analysis of desmosterol, cholesterol and cholesterol sulfate in semen by high-performance liquid chromatography. J Chromatogr A 2001; 935: 259-65.

64 Connor WE, Lin DS, Wolf DP, Alexander M. Uneven distribution of desmosterol and docosahexaenoic acid in the heads and tails of monkey sperm. J Lipid Res 1998; 39: 1404-11.

65 Cross NL. Effect of cholesterol and other sterols on human sperm acrosomal responsiveness. Mol Reprod Dev 1996; 45: 212-7.

66 Nimmo MR, Cross NL. Structural features of sterols required to inhibit human sperm capacitation. Biol Reprod 2003; 68: 1308-17.

67 Sugkraroek P, Kates M, Leader A, Tanphaichitr N. Levels of cholesterol and phospholipids in freshly ejaculated sperm and Percoll-gradient-pelletted sperm from fertile and unexplained infertile men. Fertil Steril 1991; 55: 820-7.

68 Zalata A, Hassan A, Christophe A, Comhaire F, Mostafa T. Cholesterol and desmosterol in two sperm populations separated on Sil-Select gradient. Int J Androl 2010; 33: 528-35.

69 Wathes DC, Abayasekara DR, Aitken RJ. Polyunsaturated fatty acids in male and female reproduction. Biol Reprod 2007; 77: 190-201.

70 Chabory E, Damon C, Lenoir A, Kauselmann G, Kern H et al. Epididymis selenoindependent glutathione peroxidase 5 maintains sperm DNA integrity in mice. $J$ Clin Invest 2009; 119: 2074-85.

71 Aitken RJ. Gpx5 protects the family jewels. J Clin Invest 2009; 119: 1849-51.

72 Jones R. Plasma membrane composition and organisation during maturation of spermatozoa in the epididymis. In: Robaire B, Hinton BT, editors. The Epididymis: From Molecules to Clinical Practice. A Comprehensive Survey of the Efferent Ducts, the Epididymis and the Vas Deferens. New York: Kluwer Academic/Plenum Publishers; 2002. pp405-16.

73 Lin Y, Kan FW. Regionalization and redistribution of membrane phospholipids and cholesterol in mouse spermatozoa during in vitro capacitation. Biol Reprod 1996; 55 . $1133-46$.

74 James PS, Wolfe CA, Mackie A, Ladha S, Prentice A et al. Lipid dynamics in the plasma membrane of fresh and cryopreserved human spermatozoa. Hum Reprod 1999; 14 : 1827-32.

75 Christova Y, James PS, Cooper TG, Jones R. Lipid diffusion in the plasma membrane of mouse spermatozoa: changes during epididymal maturation, effects of $\mathrm{pH}$, osmotic pressure, and knockout of the c-ros gene. J Androl 2002; 23: 384-92.

76 Christova Y, James P, Mackie A, Cooper TG, Jones R. Molecular diffusion in sperm plasma membranes during epididymal maturation. Mol Cell Endocrinol 2004; 216 : 41-6.

77 Selvaraj V, Asano A, Buttke DE, McElwee JL, Nelson JL et al. Segregation of micronscale membrane sub-domains in live murine sperm. J Cell Physiol 2006; 206: 63646.

78 Selvaraj V, Asano A, Buttke DE, Sengupta P, Weiss RS et al. Mechanisms underlying the micron-scale segregation of sterols and $\mathrm{G}_{\mathrm{M} 1}$ in live mammalian sperm. J Cell Physiol 2009; 218: 522-36.

79 Asano A, Selvaraj V, Buttke DE, Nelson JL, Green KM et al. Biochemical characterization of membrane fractions in murine sperm: identification of three distinct sub-types of membrane rafts. J Cell Physiol 2009; 218: 537-48.

80 Travis AJ, Kopf GS. The role of cholesterol efflux in regulating the fertilization potential of mammalian spermatozoa. J Clin Invest 2002; 110: 731-6.

81 Flesch FM, Gadella BM. Dynamics of the mammalian sperm plasma membrane in the process of fertilization. Biochim Biophys Acta 2000; 1469: 197-235.

82 Hermo L, Jacks D. Nature's ingenuity: bypassing the classical secretory route via apocrine secretion. Mol Reprod Dev 2002; 63: 394-410.

83 Gliozzi TM, Zaniboni L, Maldjian A, Luzi F, Maertens L et al. Quality and lipid composition of spermatozoa in rabbits fed DHA and vitamin $\mathrm{E}$ rich diets. Theriogenology 2009; 71: 910-9.

84 Mitre R, Cheminade C, Allaume P, Legrand P, Legrand AB. Oral intake of shark liver oil modifies lipid composition and improves motility and velocity of boar sperm. Theriogenology 2004; 62: 1557-66.

85 Rooke JA, Shao CC, Speake BK. Effects of feeding tuna oil on the lipid composition of pig spermatozoa and in vitro characteristics of semen. Reproduction 2001; 121: 315-22.

86 Cerolini S, Zaniboni L, Maldjian A, Gliozzi T. Effect of docosahexaenoic acid and alpha-tocopherol enrichment in chicken sperm on semen quality, sperm lipid composition and susceptibility to peroxidation. Theriogenology 2006; 66: 877-86.

87 Aumuller G, Renneberg H, Schiemann PJ, Wilhelm B, Seitz J et al. The role of apocrine released proteins in the post-testicular regulation of human sperm function. Adv Exp Med Biol 1997; 424: 193-219.

88 Frenette G, Legare C, Saez F, Sullivan R. Macrophage migration inhibitory factor in the human epididymis and semen. Mol Hum Reprod 2005; 11: 575-82. 
89 Gatti JL, Metayer S, Belghazi M, Dacheux F, Dacheux JL. Identification, proteomic profiling, and origin of ram epididymal fluid exosome-like vesicles. Biol Reprod 2005; 72: $1452-65$

90 Fornes WM, Sosa MA, Bertini F, Burgos MH. Vesicles in rat epididymal fluid. Existence of two populations differing in ultrastructure and enzymatic composition. Andrologia 1995; 27: 233-7

91 Yanagimachi R, Kamiguchi Y, Mikamo K, Suzuki F, Yanagimachi H. Maturation of spermatozoa in the epididymis of the Chinese hamster. Am J Anat 1985; 172: 317-30.

92 Frenette G, Sullivan R. Prostasome-like particles are involved in the transfer of P25b from the bovine epididymal fluid to the sperm surface. Mol Reprod Dev 2001; 59: 115-21.
93 Sullivan R, Frenette G, Girouard J. Epididymosomes are involved in the acquisition of new sperm proteins during epididymal transit. Asian J Androl 2007; 9: 483-91.

94 Girouard J, Frenette G, Sullivan R. Compartmentalization of proteins in epididymosomes coordinates the association of epididymal proteins with the different functional structures of bovine spermatozoa. Biol Reprod 2009; 80: 96572.

95 Moskovtsev SI, Jarvi K, Legare C, Sullivan R, Mullen JB. Epididymal P34H protein deficiency in men evaluated for infertility. Fertil Steril 2007; 88: 1455-7.

96 Boue F, Sullivan R. Cases of human infertility are associated with the absence of P34H an epididymal sperm antigen. Biol Reprod 1996; 54: 1018-24. 